\title{
Group analysis and some exact solutions for the thermal boundary layer
}

\author{
P. Barrera ${ }^{1} \&$ T. Brugarino ${ }^{2}$ \\ ${ }^{1}$ Dipartimento di Meccanica, Università di Palermo, \\ Facoltà d'Ingegneria, Palermo, Italia \\ ${ }^{2}$ Dipartimento di Metodi e Modelli Matematici, Università di Palermo, \\ Facoltà d'Ingegneria, Palermo, Italia
}

\begin{abstract}
We perform the group analysis of the thermal boundary layer in laminar flow. We obtain the classification of the solutions in terms of the asymptotic velocity. Some solutions of the boundary layer equations, for some distributions of outer flow velocity, are obtained also.
\end{abstract}

\section{Introduction}

It is very important to have the similarity solutions for the partial differential equations for the flow field near a body in a fluid flow. Generally the solutions of these equations are obtained by means of dimensional analysis which is a particular case of the group analysis. This is based on the theory of S. Lie developed more than one hundred years ago in order to have solutions of ordinary and partial, linear and non linear differential equations [1-6].

Considering systems of partial differential equations containing an arbitrary number of dependent and independent variables, the group analysis provides similarity solutions reducing the original system to a system with a reduced number of independent variables [7-9].

Now we turn our attention to the group analysis of the equations of the thermal boundary layer for some particular cases.

\section{Group analysis}

We show in brief the theory of one-parameter Lie groups of transformations for a partial differential equation in which the number of independent variables is equal 
to two. We can generalize the results to systems of partial differential equations containing an arbitrary number of dependent and independent variables.

Consider the partial differential equation (dependent variable $c$, independent variables $x$ and $t$ ) of second order

$$
F\left(c, x, t, c_{x}, c_{t}, c_{x x}, c_{x t}, c_{t t}\right)=0
$$

In what follows $c_{x}$ denotes $\frac{\partial c}{\partial x}, c_{t}$ denotes $\frac{\partial c}{\partial t}, \ldots$

Consider an one-parameter $(\epsilon)$ group of transformations:

$$
\left\{\begin{aligned}
c^{*} & =c^{*}(x, t, c ; \epsilon) \\
x^{*} & =x^{*}(x, t, c ; \epsilon) \\
t^{*} & =t^{*}(x, t, c ; \epsilon)
\end{aligned}\right.
$$

When $\epsilon=0$ the (2) correspond to identical transformation

$$
\left\{\begin{array}{l}
c^{*}=c \\
t^{*}=t \\
x^{*}=x
\end{array}\right.
$$

Expanding the (2) about the identity $\epsilon=0$ we obtain

$$
\left\{\begin{array}{l}
c^{*}=c+\epsilon \gamma(x, t, c)+O\left(\epsilon^{2}\right) \\
x^{*}=x+\epsilon \xi(x, t, c)+O\left(\epsilon^{2}\right) \\
t^{*}=t+\epsilon \tau(x, t, c)+O\left(\epsilon^{2}\right)
\end{array}\right.
$$

where $\gamma, \xi$ and $\tau$ are the infinitesimal generators of the transformations (2).

The $c_{x^{*}}^{*}, c_{t^{*}}^{*}, \ldots$ are the transformed derivatives determined from (2)

$$
\left\{\begin{array}{l}
c_{x^{*}}^{*}=c_{x^{*}}^{*}\left(x, t, c, c_{x}, c_{t} ; \epsilon\right) \\
c_{t^{*}}^{*}=c_{t^{*}}^{*}\left(x, t, c, c_{x}, c_{t} ; \epsilon\right) \\
c_{x^{*} x^{*}}^{*}=c_{x^{*} x^{*}}^{*}\left(x, t, c, c_{x}, c_{t}, c_{x x}, c_{x t}, c_{t t} ; \epsilon\right) \\
c_{x^{*} t^{*}}^{*}=c_{x^{*} x^{*}}^{*}\left(x, t, c, c_{x}, c_{t}, c_{x x}, c_{x t}, c_{t t} ; \epsilon\right) \\
c_{t^{*} t^{*}}^{*}=c_{x^{*} x^{*}}^{*}\left(x, t, c, c_{x}, c_{t}, c_{x x}, c_{x t}, c_{t t} ; \epsilon\right)
\end{array}\right.
$$

In similar way for the (3) we have

$$
\left\{\begin{array}{lll}
c_{x^{*}}^{*} & =c_{x}+\epsilon\left[c_{x}\right]+O\left(\epsilon^{2}\right) \\
c_{t^{*}}^{*} & =c_{t}+\epsilon\left[c_{t}\right]+O\left(\epsilon^{2}\right) \\
c_{x^{*} x^{*}}^{*} & =c_{x x}+\epsilon\left[c_{x x}\right]+O\left(\epsilon^{2}\right) \\
c_{x^{*} t^{*}}^{*} & c_{x t}+\epsilon\left[c_{x t}\right]+O\left(\epsilon^{2}\right) \\
c_{t^{*} t^{*}}^{*} & c_{t t}+\epsilon\left[c_{t t}\right]+O\left(\epsilon^{2}\right)
\end{array}\right.
$$

where $\left[c_{x}\right],\left[c_{t}\right],\left[c_{x x}\right]$ are the infinitesimal generators of the transformed derivatives determined from the (2). 
Because the (2) determine the (4), the transformed derivatives $\left[c_{x}\right],\left[c_{t}\right],\left[c_{x x}\right]$, $\left[c_{x t}\right]$ and $\left[c_{t t}\right]$ can be expressed in terms of $\gamma, \xi, \tau$.

We have for example

$$
\left[c_{x}\right]=\frac{D \gamma}{D x}-\frac{\partial c}{\partial x} \frac{D \xi}{D x}-\frac{\partial c}{\partial t} \frac{D \tau}{D x}
$$

where $\frac{D}{D x}$ is the total derivative. In a similar way we proceed for $\left[c_{t}\right]$.

Higher order transformed derivatives can be derived from recurrence formulas. The differential equation (1) is invariant under the group of transformations (2) if

$$
\begin{aligned}
& F\left(c, x, t, c_{x}, c_{t}, c_{x x}, c_{x t}, c_{t t}\right)= \\
& F\left(c+\epsilon \gamma+\cdots, x+\epsilon \xi+\cdots, t+\epsilon \tau+\cdots, c_{x}+\epsilon\left[c_{x}\right]+\cdots, c_{t}+\epsilon\left[c_{t}\right]+\cdots,\right. \\
& \left.c_{x x}+\epsilon\left[c_{x x}\right]+\cdots, c_{x t}+\epsilon\left[c_{x t}\right]+\cdots, c_{t t}+\epsilon\left[c_{t t}\right]+\cdots\right)
\end{aligned}
$$

Expanding the right member of the (5), we have

$$
\begin{aligned}
& F\left(c, x, t, c_{x}, c_{t}, c_{x x}, c_{x t}, c_{t t}\right)=F\left(c, x, t, c_{x}, c_{t}, c_{x x}, c_{x t}, c_{t t}\right)+ \\
& \epsilon\left(F_{c} \gamma+F_{x} \xi+F_{t} \tau+F_{c_{x}}\left[c_{x}\right]+F_{c_{t}}\left[c_{t}\right]+F_{c_{x x}}\left[c_{x x}\right]+F_{c_{x t}}\left[c_{x t}\right]+F_{c_{t t}}\left[c_{t t}\right]\right)+\cdots
\end{aligned}
$$

and so the invariance condition of the equation is

$$
F_{c} \gamma+F_{x} \xi+F_{t} \tau+F_{c_{x}}\left[c_{x}\right]+F_{c_{t}}\left[c_{t}\right]+F_{c_{x x}}\left[c_{x x}\right]+F_{c_{x t}}\left[c_{x t}\right]+F_{c_{t t}}\left[c_{t t}\right]=0
$$

If the solution is invariant under the group of transformations, the solution must map into itself, i.e.

$$
c^{*}=c\left(x^{*}, t^{*}\right)=c(x+\epsilon \xi, t+\epsilon \tau)
$$

In terms of the transformation functions, eq. (6) can be written as.

$$
c(x+\epsilon \xi, t+\epsilon \tau)=c(z, t)+\epsilon \gamma(z, t, c)+O\left(\epsilon^{2}\right)
$$

Expanding the left-hand side of eq. (7) and equating the coefficients of $\epsilon$, we get

$$
\gamma=c_{x} \xi+c_{t} \tau
$$

The eq. (8) is the invariant surface condition. We can call the eq. (7) also as invariance condition of the solution. The general solution of eq. (7) is obtained by solving the characteristic equation

$$
\frac{\mathrm{d} x}{\xi}=\frac{\mathrm{d} t}{\tau}=\frac{\mathrm{d} c}{\gamma}
$$

In principle, the general solution of eq. (8) can be found. It involves two constants, one becomes the independent variable $\xi(c, x, t)$, called the similarity variable and the other is the dependent variable $f(\xi)$. We obtain the similarity solution

$$
c=\mathcal{F}(t, x, \xi, f(\xi))
$$

with the dependence of $\mathcal{F}$ on $x, t$ and the arbitrary function $f(\xi)$ known explicitly. Substitution of (10) into (1) results an ordinary differential equation for the function $f(\xi)$. 
The application of the group analysis, also for modest systems of differential equations, involves long and tedious computations. Symbolic packages are very useful for such computations.

\section{Thermal boundary layer equations}

If the properties $c_{p}, \mu$ and $k$ can be assumed independent of temperature and the pressure gradient in the $x$-direction is different from zero, the thermal boundary layer equations for two-dimensional incompressible steady fluid flow are (see Schlichting [10], Schlichting and Gersten [11] and Rosenhead [12]):

$$
\left\{\begin{array}{l}
\frac{\partial \Psi}{\partial y} \frac{\partial^{2} \Psi}{\partial x \partial y}-\frac{\partial \Psi}{\partial x} \frac{\partial^{2} \Psi}{\partial y^{2}}=\nu \frac{\partial^{3} \Psi}{\partial y^{3}}+U(x) \frac{\mathrm{d} U(x)}{\mathrm{d} x} \\
\frac{\partial \Psi}{\partial y} \frac{\partial T}{\partial x}-\frac{\partial \Psi}{\partial x} \frac{\partial T}{\partial y}=\frac{k}{\rho c_{p}} \frac{\partial^{2} T}{\partial y^{2}}+\frac{\nu}{c_{p}}\left(\frac{\partial^{2} \Psi}{\partial y^{2}}\right)^{2}-\frac{1}{c_{p}} \frac{\partial \Psi}{\partial y} U(x) \frac{\mathrm{d} U(x)}{\mathrm{d} x}
\end{array}\right.
$$

where:

- $\Psi(x, y) \Rightarrow$ Stream function $\left(u=\frac{\partial \Psi}{\partial y}, v=-\frac{\partial \Psi}{\partial x}\right)$;

- $U(x) \Rightarrow$ Outer flow velocity;

- $T(x, t) \Rightarrow$ Fluid temperature;

- $\nu \Rightarrow$ Kinematic viscosity;

- $k \Rightarrow$ Thermal conductivity;

- $c_{p} \Rightarrow$ Specific heat at constant pressure;

- $\rho \Rightarrow$ Density.

The boundary conditions for eqs. (11) are:

$$
\left\{\begin{array}{l}
u=v=0 \text { at } y=0 \\
u=U(x) \text { as } y \rightarrow \infty \\
T=T_{w} \text { at } y=0 \\
T=T_{\infty} \text { as } y \rightarrow \infty
\end{array}\right.
$$

where :

- $T_{w} \Rightarrow$ Wall temperature

- $T_{\infty} \Rightarrow$ Free stream temperature

Consider the following one-parameter Lie group of transformations in order to leave invariant the eqs. (11):

$$
\left\{\begin{array}{l}
x^{\prime}=x+\epsilon \xi^{1}(x, y, \Psi, T)+\cdots \\
y^{\prime}=y+\epsilon \xi^{2}(x, y, \Psi, T)+\cdots \\
\Psi^{\prime}=\Psi+\epsilon \eta(x, y, \Psi, T)+\cdots \\
T^{\prime}=T+\epsilon \tau(x, y, \Psi, T)+\cdots
\end{array}\right.
$$

The invariance conditions for eqs. (11) give us the following equations for the infinitesimal generators of the transformations (12) and for the free-stream flow 
velocity $U(x)$ :

$$
\left\{\begin{array}{l}
\xi_{y}^{1}=\xi_{\Psi}^{1}=\xi_{T}^{1}=\xi_{\Psi}^{2}=\xi_{T}^{2}=\eta_{x}=\eta_{y}=\eta_{T}=\tau_{y}=\eta_{\Psi}=0 \\
\xi_{y y}^{2}=\xi_{x y}^{2}=\eta_{\Psi \Psi}=\tau_{T T}=0 \\
\xi_{y}^{2}+\eta_{\Psi}-\xi_{x}^{1}=0 \\
2\left(\xi_{y}^{2}-\eta_{\Psi}\right)+\tau_{T}=0 \\
-U U_{x x} \xi^{1}-3 U U_{x} \xi_{y}^{2}+U U_{x} \eta_{\Psi}-U_{x}^{2} \xi^{2}=0 \\
-U U_{x x} \xi^{1}-U U_{x} \xi_{y}^{2}-U U_{x} \eta_{\Psi}+\tau_{t} U U_{x}-\xi^{1} U_{x}^{2}+\frac{\tau_{x}}{\nu}=0
\end{array}\right.
$$

The system (13) admits the following solution:

$$
\left\{\begin{array}{l}
\xi^{1}=(A+C) x+D \\
\xi^{2}=A y+M(x) \\
\eta_{=} C \Psi+B \\
\tau=2(C-A) T+R
\end{array}\right.
$$

where $A, B, C, D, R$ are arbitrary constants and $M(x)$ is an arbitrary but regular function of $x$. For the free stream flow velocity we obtain the following differential equation [13]:

$$
(3 A-C) \frac{\mathrm{d} U^{2}}{\mathrm{~d} x}+((A+C) x+D) \frac{\mathrm{d}^{2} U^{2}}{\mathrm{~d} x^{2}}=0
$$

We performed the calculations of the generators of transformations group on a PC using the REDUCE and MATHEMATICA packages.

Each of the constants $A, B, C, D, R$ and the function $U(x)$ can be taken in turn to generate a similarity form for the solution.

\section{Similarity solutions}

Let us look at particular similarity solutions.

$$
\text { 4.1 } C \neq A=1, D=B=R=M=0, A+C \neq 0
$$

The characteristic equations are:

$$
\frac{\mathrm{d} x}{(1+C) x}=\frac{\mathrm{d} y}{y}=\frac{\mathrm{d} \Psi}{C \Psi}=\frac{\mathrm{d} T}{2(C-1) T}
$$

It is possible to have the following invariants

$$
\left\{\begin{array}{l}
I_{1}=y x^{-\frac{1}{1+C}} \\
I_{2}=\Psi x^{-\frac{C}{1+C}} \\
I_{3}=T x^{2 \frac{1-C}{1+C}}
\end{array}\right.
$$


The invariant $I_{1}=y x^{-\frac{1}{1+C}}$ is the similarity variable $\xi$ and the similarity solutions are:

$$
\left\{\begin{array}{l}
\Psi=x^{\frac{C}{1+C}} f\left(y x^{-\frac{1}{1+C}}\right) \\
T=x^{-2 \frac{1-C}{1+C}} \theta\left(y x^{-\frac{1}{1+C}}\right)
\end{array}\right.
$$

The functions $f(\xi)$ and $\theta(\xi)$ satisfy the following equations obtained from eqs. (11)

$$
\left\{\begin{array}{l}
\frac{1-C}{1+C} f^{\prime 2}+\frac{C}{1+C} f f^{\prime \prime}+\nu f^{\prime \prime \prime}+\alpha=0 \\
2 \frac{C-1}{1+C} f^{\prime} \theta-\frac{C}{1+C} f \theta^{\prime}-\frac{k}{\rho c_{p}} \theta^{\prime \prime}-\frac{\nu}{c_{p}} f^{\prime \prime 2}+\frac{\alpha}{c_{p}} f^{\prime}=0
\end{array}\right.
$$

where $\alpha$ is a constant.

The free stream flow velocity, considering eq. (14), is solution of the equation:

$$
U U_{x}=\alpha x^{\frac{C-3}{C+1}}
$$

We have

$$
U^{2}=U_{l}^{2}\left(\frac{x}{l}\right)^{2 n}+U_{0}^{2}
$$

if:

$$
C=\frac{1+n}{1-n} \quad \text { and } \quad U_{l}^{2}=n \alpha l^{2 n} \quad(n \neq 1)
$$

The eq. (19) correspond to the flow past a wedge, in the neighborhood of the leading edge [11].

In particular, if $C=0(n=-1)$ we obtain:

$$
U^{2}=U_{l}^{2}\left(\frac{l}{x}\right)^{2}+U_{0}^{2}
$$

(flow in converging or diverging channel [11]).

\subsubsection{Flow past a wedge, in the neighborhood of the leading edge} If $U_{0}=0$, we have:

$$
U=U_{l}\left(\frac{x}{l}\right)^{n}
$$

In this case the component $u$ of the velocity is:

$$
u=x^{n} f\left(y x^{\frac{n-1}{2}}\right)
$$

and the boundary conditions become

$$
\begin{cases}f(0) & =f^{\prime}(0)=0 \\ f^{\prime}(\infty) & =\frac{U_{l}}{l^{n}} \\ \theta(0) & =\theta_{0} \\ \theta(\infty) & =0\end{cases}
$$


If we assume $C=\frac{1}{2}$, then $n=-\frac{1}{3}$ [11]. The first of eqs. (18) become:

$$
\frac{1}{3} f^{\prime 2}+\frac{1}{3} f f^{\prime \prime}+\nu f^{\prime \prime \prime}-3 U_{l}^{2} l^{\frac{2}{3}}=0
$$

The eq. (20) is analytically integrable. Integrating the equation twice and using the boundary conditions, we obtain:

$$
K \xi+\frac{1}{6} f^{2}+\nu f^{\prime}-\frac{3}{2} U_{l}^{2} \xi^{2} l^{\frac{2}{3}}=0
$$

The eq. (21) is a Riccati equation [14] and its solution is:

$$
\begin{gathered}
f(\xi)=\left\{3 \sqrt{2 l U_{l}^{3} \nu}\left(K^{2}-18 l U_{l}^{3} \nu\right) C_{1} \mathrm{H} \frac{A^{2}}{36 l U_{l}^{3} \nu}-\frac{3}{2}\left(\frac{3 l^{\frac{2}{3}} U_{l}^{2} \xi-K}{3 \sqrt{2 l U_{l}^{3} \nu}}\right)+\left(K-3 l^{\frac{2}{3}} U_{l}^{2} \xi\right) \times\right. \\
{\left[18 l U_{l}^{3} \nu\left(C_{1} \mathrm{H} \frac{K^{2}}{36 l U_{l}^{3} \nu}-\frac{1}{2}\left(\frac{3 l^{\frac{2}{3}} U_{l}^{2} \xi-K}{3 \sqrt{2 l U_{l}^{3} \nu}}\right)+{ }_{1} \mathrm{~F}_{1}\left(\frac{\left(K-3 l^{\frac{2}{3}} U_{l}^{2} \xi\right)^{2}}{18 l U_{l}{ }^{3} \nu} ; \frac{1}{4}-\frac{K^{2}}{72 l U_{l}{ }^{3} \nu}, \frac{1}{2}\right)\right)+\right.} \\
\left.\left.\left(K^{2}-18 l U_{l}^{3} \nu\right){ }_{1} \mathrm{~F}_{1}\left(\frac{\left(K-3 l^{\frac{2}{3}} U_{l}^{2} \xi\right)^{2}}{18 l U_{l}{ }^{3} \nu} ; \frac{5}{4}-\frac{K^{2}}{72 l U_{l}{ }^{3} \nu}, \frac{3}{2}\right)\right]\right\} \times \\
\left\{18 l^{\frac{4}{3}} U_{l}^{4} \nu\left[C_{1} \mathrm{H} \frac{K^{2}}{36 l U_{l}^{3} \nu}-\frac{1}{2}\left(\frac{3 l^{\frac{2}{3}} U_{l}^{2} \xi-K}{3 \sqrt{2 l U_{l}^{3} \nu}}\right)+{ }_{1} \mathrm{~F}_{1}\left(\frac{\left(K-3 l^{\frac{2}{3}} U_{l}{ }^{2} \xi\right)^{2}}{18 l U_{l}^{3} \nu} ; \frac{1}{4}-\frac{K^{2}}{72 l U_{l}{ }^{3} \nu}, \frac{1}{2}\right)\right]\right\}^{-1}
\end{gathered}
$$

where ${ }_{1} \mathrm{~F}_{1}(-;-,-)$ is the Kummer confluent hypergeometric function and $\mathrm{H}_{-}(-)$the Hermite function; the constant $K$ and $C_{1}$ are determined by boundary conditions.

\subsubsection{Flow converging or diverging channel}

If $C=0$ and $U_{0}=0$, then we have $n=-1$ :

$$
U=U_{l} \frac{l}{x}
$$

The component $u$ of the velocity is:

$$
u=x^{-1} f^{\prime}\left(y x^{-1}\right)
$$

the boundary conditions become

$$
\left\{\begin{array}{l}
f(0)=f^{\prime}(0)=0 \\
f^{\prime}(\infty)=U_{l} l \\
\theta(0)=\theta_{0} \\
\theta(\infty)=0
\end{array}\right.
$$

The first of eqs. (18) becomes:

$$
f^{\prime 2}+\nu f^{\prime \prime \prime}-U_{l}^{2} l^{2}=0
$$

The eq. (22) is analytically integrable. 
The transformation: $h(\xi)=f^{\prime}(\xi)$ reduces eq. (22) to the equation:

$$
h^{2}+\nu h^{\prime \prime}-U_{l}^{2} l^{2}=0
$$

The eq. (23) is a Painlevé type equation (see [15]); its solution is expressed in term of Weierstrass elliptic function $\wp$. The equation eq. (23) is equivalent to

$$
\left(h^{\prime}\right)^{2}=\frac{2}{\nu}\left(l U_{l}\right)^{2} h-\frac{2}{3 \nu} h^{3}-H
$$

The substitution $h(\xi)=-6 \nu z(\xi)$ leads to the equation:

$$
\left(z^{\prime}\right)^{2}=4 z^{3}-\frac{\left(l U_{l}\right)^{2}}{3 \nu^{2}} z-H
$$

The solution of this equation is:

$$
z(\xi)=\wp\left(\xi-C_{1} ; \frac{\left(l U_{l}\right)^{2}}{3 \nu^{2}}, H\right)
$$

where $\wp\left(-; g_{2}, g_{3}\right)$ is the Weierestrass elliptic function.

The solution of eq. (22) is therefore:

$$
f(\xi)=6 \nu \zeta\left(\xi-C_{1} ; \frac{\left(l U_{l}\right)^{2}}{3 \nu^{2}}, H\right)+K
$$

where $\zeta\left(-; g_{2}, g_{3}\right)\left(\zeta^{\prime}=-\wp\right)$ is the Weierestrass $\zeta$-function (see [16]), the constant $H, C_{1}$ and $K$ are determined by boundary conditions. It is worthy of remark that for $\Delta=g_{2}^{3}-27 g_{3}^{2}=0$ and for particular values of $g_{2}$ and $g_{3}$, the Weierestrass $\wp$-function degenerates to the hyperbolic function [16]; in this case we have the Pohlhausen solution [10].

$$
\text { 4.2 } A=D=B=R=0, M \neq 0, C \neq 0
$$

From the characteristic equations we have:

$$
\left\{\begin{array}{c}
\Psi=x f(y-h(x)) \\
T=x^{2} \theta(y-h(x))
\end{array}\right.
$$

where $h(x)$ is an arbitrary function. The similarity equations are:

$$
\left\{\begin{array}{l}
f^{2}-f f^{\prime \prime}-\nu f^{\prime \prime \prime}-\alpha=0 \\
2 f^{\prime} \theta-f \theta^{\prime}-\frac{k}{\rho c_{p}} \theta^{\prime \prime}-\frac{\nu}{c_{p}} f^{\prime \prime 2}+\frac{\alpha}{c_{p}} f^{\prime}=0
\end{array}\right.
$$

where $\alpha$ is a constant. If we put

$$
\alpha=\frac{U_{l}^{2}}{l^{2}}
$$


the free stream flow velocity is:

$$
U^{2}=\frac{U_{l}^{2}}{l^{2}} x^{2}+U_{0}^{2}
$$

If

$$
U_{0}=0
$$

we have

$$
U=U_{l} \frac{x}{l}
$$

This is the flow with forward or rear stagnation point [12]. In this case the component $u$ of the velocity is $(h(x)=0)$ :

$$
u=x f^{\prime}(y)
$$

and the boundary conditions become

$$
\begin{cases}f(0) & =f^{\prime}(0)=0 \\ f^{\prime}(\infty) & =\frac{U_{l}}{l} \\ \theta(0) & =\theta_{0} \\ \theta(\infty) & =0\end{cases}
$$

4.3 $A+C=B=R=M=0, D=1$

In this case:

$$
\left\{\begin{aligned}
\Psi & =\mathrm{e}^{C x} f\left(y \mathrm{e}^{C x}\right) \\
T & =\mathrm{e}^{4 C x} \theta\left(y \mathrm{e}^{C x}\right)
\end{aligned}\right.
$$

The similarity solutions are

$$
\left\{\begin{array}{l}
2 C f^{\prime 2}-C f f^{\prime \prime}-\nu f^{\prime \prime \prime}-\alpha=0 \\
4 C f^{\prime} \theta-C f \theta^{\prime}-\frac{k}{\rho c_{p}} \theta^{\prime \prime}-\frac{\nu}{c_{p}} f^{\prime \prime 2}+\frac{\alpha}{c_{p}} f^{\prime}=0
\end{array}\right.
$$

where $\alpha$ is a constant. The free stream flow velocity is:

$$
U^{2}=U_{l}^{2}+\frac{\alpha}{2 C}\left(\mathrm{e}^{4 C x}-\mathrm{e}^{4 C l}\right)
$$

If we assume:

$$
U_{l}=\frac{\alpha}{2 C} \mathrm{e}^{4 C l}
$$

then

$$
U=\sqrt{\frac{\alpha}{2 C}} \mathrm{e}^{2 C x}
$$

In this case [10] the component $u$ of the velocity is:

$$
u=\mathrm{e}^{2 C x} f^{\prime}\left(y \mathrm{e}^{C x}\right)
$$


and the boundary conditions become

$$
\left\{\begin{array}{l}
f(0) \\
f^{\prime}(\infty)=f^{\prime}(0)=0 \\
\theta(0)=\theta_{0} \\
\theta(\infty)=0
\end{array}\right.
$$

4.4 $D=B=R=M=0, A=C=1, U=$ constant

The stream function and the temperature are:

$$
\left\{\begin{array}{l}
\Psi=x^{\frac{1}{2}} f\left(y x^{-\frac{1}{2}}\right) \\
T=\theta\left(y x^{-\frac{1}{2}}\right)
\end{array}\right.
$$

The functions $f$ and $\theta$ satisfy the following equations:

$$
\left\{\begin{array}{l}
\frac{1}{2} f f^{\prime \prime}+\nu f^{\prime \prime \prime}=0 \\
\frac{1}{2} f \theta^{\prime}+\frac{k}{\rho c_{p}} \theta^{\prime \prime}+\frac{\nu}{c_{p}} f^{\prime \prime 2}=0
\end{array}\right.
$$

This solution corresponds to semi-infinity flat plat (Blasius solution).

\section{Conclusions}

Therefore we can be sure that the group analysis, compared to dimensional analysis or ad hoc position, enable to have with methodical work similarity solutions: all the solutions derived in standard text [10], [11] and [12] are therefore group invariant solutions.

\section{References}

[1] S. Lie, Theorie der Transformation Gruppen, Chelsea, 1974.

[2] G. W. Bluman and J. D. Cole, Similarity Methods for Differential Equations, Springer-Verlag, 1974.

[3] W. F. Ames, Nonlinear Partial Differential Equations, Academic Press, 1965-1972.

[4] L. V. Ovsiannikov, Group analysis of differential equations, Academic Press, 1982.

[5] G. W. Bluman and S. Kumei, Symmetries and Differential Equations, Springer-Verlag, 1989.

[6] P. O. Olver, Applications of Lie Groups to Differential Equations, Springer, 1993.

[7] P. Barrera and T. Brugarino, Similarity Solutions of the Generalized Kadomtsev Petviashvili-Burgers Equations, Il Nuovo Cimento B, 92, 2, (1986), 142 156. 
[8] P. Barrera and T. Brugarino, Group analysis and similarity solutions of the compressible boundary layer equations, Meccanica, 24 (1989), 211-215.

[9] P. Barrera, T. Brugarino and L. Pignato, Solutions for a Diffusion Process in non Homogeneous Media, Il Nuovo Cimento B, 116, 8, (2001), 951-958.

[10] H. Schlichting, Boundary Layer Theory, Mc Graw-Hill, 1968.

[11] H. Schlichting and K. Gersten Boundary Layer Theory, Springer-Verlag, 2000.

[12] L. Rosenhead, Laminar Boundary Layery Oxford University Press, 1963.

[13] P. Barrera, T. Brugarino, Analisi gruppale delle equazioni dello strato limite, IX Congresso AIDAA, 1987.

[14] A. D. Polyanin and V. F. Zaitsev Handbook of Exact Solutions for Ordinary Differential Equations, CRC Press, 1995.

[15] H. T. Davis, Introduction to Nonlinear Differential and Integral Equations, Dover Publications, 1962.

[16] M. Abramowitz and I. Stegun, Handbook of Mathematical Functions, Dover Publications, 1972. 